\title{
BMJ Open Interpregnancy intervals and child development at age 5: a population data linkage study
}

\author{
Gursimran Kaur Dhamrait (D) ,1,2 Catherine Louise Taylor (D) ,1,3 Gavin Pereira (D) ${ }^{1,4,5}$
}

To cite: Dhamrait GK, Taylor CL, Pereira G. Interpregnancy intervals and child development at age 5 : a population data linkage study. BMJ Open 2021;11:e045319. doi:10.1136/ bmjopen-2020-045319

\section{- Prepublication history and} additional materials for this paper is available online. To view these files, please visit the journal online (http://dx.doi org/10.1136/bmjopen-2020045319).

Received 28 September 2020 Revised 27 January 2021 Accepted 05 March 2021

Check for updates

(C) Author(s) (or their employer(s)) 2021. Re-use permitted under CC BY-NC. No commercial re-use. See rights and permissions. Published by BMJ.

${ }^{1}$ Telethon Kids Institute, University of Western Australia, Perth, Western Australia, Australia

${ }^{2}$ School of Population and Global Health, University of Western Australia, Perth, Western Australia, Australia

${ }^{3}$ Centre for Child Health Research, University of Western Australia, Perth, Western Australia, Australia

${ }^{4}$ Curtin School of Population Health, Curtin University, Perth, Western Australia, Australia

${ }^{5}$ Centre for Fertility and Health (CeFH), Norwegian Institute of Public Health, Oslo, Norway

Correspondence to Gursimran Kaur Dhamrait; gursimran.dhamrait@ telethonkids.org.au

\section{ABSTRACT}

Objective To investigate the associations between interpregnancy intervals (IPIs) and developmental vulnerability in children's first year of full-time school (age 5).

Design Retrospective cohort study using logistic regression. ORs were estimated for associations with IPIs with adjustment for child, parent and community sociodemographic variables.

Setting Western Australia (WA), 2002-2015.

Participants 34574 WA born singletons with a 2009, 2012 or 2015 Australian Early Development Census (AEDC) record.

Main outcome measure The AEDC measures child development across five domains; Physical Health and Wellbeing, Social Competence, Emotional Maturity, Language and Cognitive Skills (school-based) and Communication Skills and General Knowledge. Children with scores $<10$ th percentile were classified as developmentally vulnerable on, one or more domains (DV1), or two or more domains (DV2).

Results $22.8 \%$ and $11.5 \%$ of children were classified as DV1 and DV2, respectively. In the adjusted models (relative to the reference category, IPIs of 18-23 months), IPIs of $<6$ months were associated with an increased risk of children being classified as DV1 (adjusted OR (aOR) 1.17, $95 \% \mathrm{Cl} 1.08$ to 1.34 ), DV2 (aOR $1.31,95 \% \mathrm{Cl} 1.10$ to 1.54 ) and an increased risk of developmental vulnerability for the domains of Physical Health and Wellbeing (aOR 1.25, $95 \% \mathrm{Cl} 1.06$ to 1.48) and Emotional Maturity (aOR 1.36, $95 \% \mathrm{Cl} 1.12$ to 1.66$)$. All IPIs longer than the reference category were associated with and increased risk of children being classified as DV1 and DV2 (aOR >1.15). IPIs of $60-119$ months and $\geq 120$ months, were associated with an increased risk of developmental vulnerability on each of the five AEDC domains, with greater odds for each domain for the longer IPI category.

Conclusions IPIs showed independent J-shaped relationships with developmental vulnerability, with short ( $<6$ months) and longer ( $\geq 24$ months) associated with increased risks of developmental vulnerability.

\section{INTRODUCTION}

Interpregnancy interval (IPI), the time from birth to the conception of the next pregnancy, has been proposed as an important modifiable risk factor for adverse birth and perinatal outcomes. ${ }^{1}$ The WHO recommends an IPI

\section{Strengths and limitations of this study}

- The study is based on a large population-level and otherwise healthy sample of singleton Australian children at the time of their first year of full-time school.

- The study is the first to examine associations between interpregnancy intervals and child developmental vulnerability in a population of healthy children in the early childhood period.

- Logistic regression analysis with the calculation of adjusted ORs was performed to explore the associations between multiple interpregnancy interval categories.

- Important social risk factors including parenting experience and/or technique, stability and quality of housing and availability of learning resources within the household could not be accounted for

- We did not have information as to whether the pregnancies were planned or unplanned and as administrative records do not include pregnancies ending before 20 weeks of gestation, we are unable to identify and account for the effect of miscarriages.

of approximately 2-3 years to reduce infant and child morbidity and mortality and these recommendations are informed by several studies which have reported a strong J-shaped relationship between various adverse birth outcomes and IPIs, with the lowest risk of adverse perinatal outcomes observed for IPIs of 18-23 months. ${ }^{1-5}$ Both shorter ( $<6$ months) and longer IPIs (>60 months) have been reported to be associated with an increased risk of adverse birth outcomes however, it is believed that the pathways governing these outcomes are different. Associations between short IPIs and adverse birth outcomes have been interpreted as evidence in support of the maternal depletion hypothesis, which proposes that short IPIs lead to insufficient recovery time from a pregnancy and the subsequent period of lactation. ${ }^{136-8}$ Associations between longer IPIs and adverse birth outcomes have been interpreted as support for the physical regression hypothesis, such that long IPIs result 
in a loss of the beneficial physiological adaptations from the previous pregnancy thus, resulting in a state which resembles a primigravida. ${ }^{9}$

There is a paucity of research investigating the effects of IPIs on children's development during the early childhood period. Studies investigating the impacts of IPIs on child development outcomes have typically focused on neurodevelopmental morbidities and disabilities such as autism spectrum disorder or attention-deficit/hyperactivity problems. ${ }^{10-13}$ In terms of school-based outcomes, studies have more readily examined the impact of adversities associated with suboptimal IPIs such as preterm birth and low birth weight. The available studies assessing the relationship between birth spacing and child development outcomes, beyond those observed at birth and in children without diagnosed developmental disabilities, have primarily examined the impact of birth intervals. ${ }^{14-18}$ To our knowledge, one study has examined the associations between IPIs and childhood development during the early and middle childhood periods for children without diagnosed developmental disabilities. ${ }^{19}$ However, the findings of this study reported no statistically significant associations between IPI duration and child development outcomes, after adjustment for a range of sociodemographic factors. ${ }^{19}$ Furthermore, existing studies have reported mixed findings for the associations between birth intervals and school performance. ${ }^{1620}$ Compared with IPIs, interdelivery intervals have an inherent bias, as this measure is conflated by the gestational length of the subsequent pregnancy. ${ }^{21}$ Thus, IPIs are a measure of sibling spacing that are not confounded by gestational age. Furthermore, the first 5 years of a child's life is recognised as a critical time for identifying and responding to developmental vulnerability. Children's developmental achievements in the early childhood period lay the foundation for success at school. School readiness is a multidimensional concept that includes the child's physical health and wellbeing, social and emotional competence, language and cognitive development and communication skills and general knowledge $^{22}$ as well as attitudes towards learning and classroom skills and behaviours. ${ }^{23}$ This study aimed to examine the association between IPIs and child developmental vulnerability in the first year of full-time school in Australia.

\section{METHODS}

\section{Data sources}

This study used anonymised individual-level data from the Midwives Notification System (MNS), which is a statutory record of all births (stillborn and live-born) in Western Australia (WA) with either a birth weight $>400$ $\mathrm{g}$ and/or a final gestational length of $\geq 20$ weeks. MNS variables were cross validated with corresponding records from WA Birth Registrations. Australian Early Development Census (AEDC) records were obtained for all available years (2009, 2012 and 2015) for all children with WA

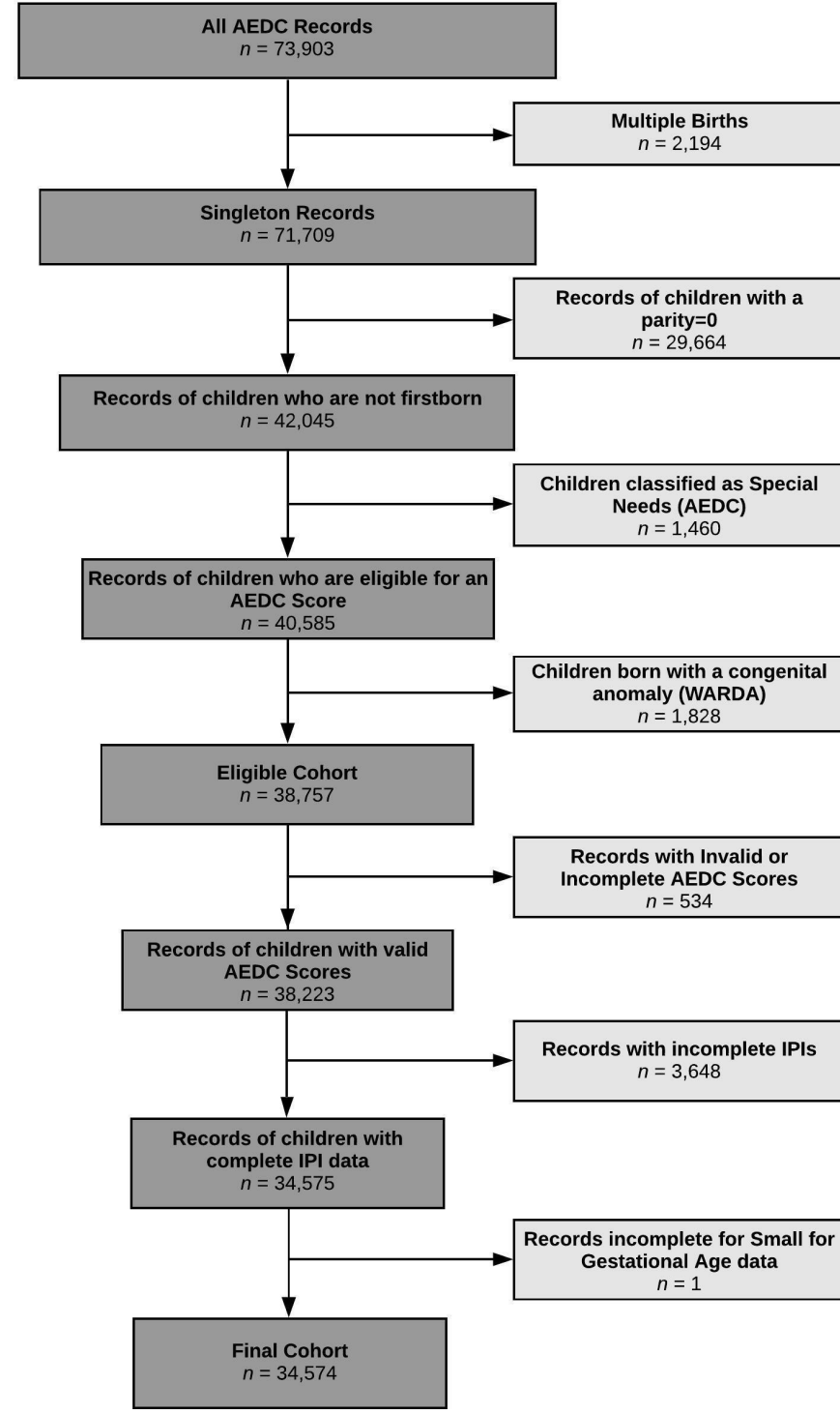

Figure 1 Eligible cohort and numbers included for analyses. AEDC, Australian Early Development Census; IPIs, Interpregnancy Intervals; WARDA, Western Australian Register of Developmental Anomalies.

birth and perinatal records. WA Register for Developmental Anomalies (WARDA) records were used to identify children with a diagnosed developmental disability. Statistical linkage of all records, by matching identifiers (eg, name, address, date of birth) common to sets of records, ${ }^{24}$ was provided by the WA Data Linkage Branch from the Department of Health WA.

\section{Study population}

The study population included all children born in WA with an AEDC record in either 2009, 2012 or 2015 $(n=73903$; figure 1$)$. Children were sequentially excluded from the study if they, (1) were from a multiple birth $(\mathrm{n}=2194(3.0 \%))$; (2) had a parity equal to zero, that is, were firstborns $(\mathrm{n}=29664(41.4 \%))$; (3) identified by their teacher as having 'special-needs' based on a diagnosed physical and/or intellectual disability $(n=1460$ $(3.5 \%))$; (4) were reported as having any congenital anomaly in WARDA $(\mathrm{n}=1828(4.5 \%))$; (5) had invalid/ 
incomplete AEDC scores ( $\mathrm{n}=534 \quad(1.4 \%))$; (6) had missing IPIs $(\mathrm{n}=3648(9.5 \%))$; or $(7)$ had missing small for gestational age data $(\mathrm{n}=1(>0.01 \%))$. The final study sample consisted of 34574 children.

\section{Patient and public involvement}

No patients were involved in the development of the research question or the outcome measures, or in the development of the plans for the design or implementation of the study.

\section{Outcome measure}

The AEDC is a national census of early childhood development spanning across five developmental domains; (1) Physical Health and Wellbeing, (2) Social Competence, (3) Emotional Maturity, (4) Language and Cognitive Skills (school-based) and (5) Communication Skills and General Knowledge. Based on the Canadian Early Development Instrument, ${ }^{25}$ the AEDC is a teachercompleted instrument collected for all children in the first year of compulsory schooling, which in WA is preprimary (the year level prior to grade 1). The AEDC is conducted every 3 years, with the first national data collection conducted in 2009. ${ }^{26}$ AEDC cut-off scores are based on the first national AEDC data collection in 2009 and apply to all AEDC data collections. ${ }^{27}$ Children who score less than the 10th percentile in a given domain are classified as 'developmentally vulnerable.' A child is classified as 'special needs' if they require special assistance because of chronic medical, physical or intellectually disabling condition. Domain scores are not calculated for those students classified as 'special needs,' as these children have already been identified as having substantial developmental needs. Across the 2009, 2012 and 2015 AEDC data collections child participation for the state of WA ranged between $98.7 \%$ and $99.6 \%{ }^{28}$ We used two summarised outcome measures; developmentally vulnerable on one or more AEDC domains (DV1), and developmentally vulnerable on two or more AEDC domains (DV2), and assessed developmental vulnerability on each AEDC domain.

\section{Exposure variables}

IPI was derived as the time between the birth of the older sibling and the estimated start of the pregnancy (birth date minus gestational age of child, measured in completed weeks of gestation) of the cohort child. In line with previous studies, ${ }^{1-4} 7$ short IPIs were classified as; $<6$ months, 6-11 months and 12-17 months, IPIs of 18-23 months formed the reference category and long IPIs were classified as; 24-59 months, 60-119 months and $\geq 120$ months.

\section{Adjustment variables}

Adjustments were made for pregnancy and birth, child and sociodemographic characteristics, selected on the basis of availability and findings of previous studies (table 1) ${ }^{29-32}$ We conducted univariate analysis for the association between the background characteristics and
Table 1 Characteristics of the study cohort

Total population

n (\%)

Characteristics

$\mathrm{n}=\mathbf{3 4 5 7 4}$

Children classified as developmentally vulnerable on 7899 (22.8) one or more AEDC domains (DV1)

Children classified as developmentally vulnerable on 3966 (11.5) two or more AEDC domains (DV2)

\begin{tabular}{|c|c|c|}
\hline \multicolumn{3}{|l|}{ Pregnancy and birth } \\
\hline \multirow{7}{*}{$\begin{array}{l}\text { Interpregnancy interval } \\
\text { (months) }\end{array}$} & $<6$ & $1703(4.9)$ \\
\hline & $6-11$ & $5226(15.1)$ \\
\hline & $12-17$ & $6451(18.7)$ \\
\hline & $18-23^{*}$ & $5311(15.4)$ \\
\hline & $24-59$ & $11531(33.4)$ \\
\hline & $60-119$ & $3517(10.2)$ \\
\hline & $\geq 120$ & $835(2.4)$ \\
\hline \multirow{2}{*}{$\begin{array}{l}\text { Maternal smoking status } \\
\text { during pregnancy }\end{array}$} & $\mathrm{No}^{*}$ & 28331 (81.9) \\
\hline & Yes & $6243(18.1)$ \\
\hline \multirow[t]{3}{*}{ Mode of delivery } & Vaginal birth* & $21654(62.6)$ \\
\hline & Caesarean birth & $10949(31.7)$ \\
\hline & All other & $1971(5.7)$ \\
\hline \multirow[t]{2}{*}{ Preterm birth } & Term* & $32488(94.0)$ \\
\hline & Preterm & $2086(6.0)$ \\
\hline \multirow[t]{2}{*}{ Small for gestational age } & $\mathrm{No}^{*}$ & $32404(93.7)$ \\
\hline & Yes & $2170(6.3)$ \\
\hline \multirow[t]{3}{*}{ Parity } & $1^{*}$ & $20065(58.0)$ \\
\hline & 2 & $9086(26.3)$ \\
\hline & $\geq 3$ & $5423(15.7)$ \\
\hline \multirow{6}{*}{$\begin{array}{l}\text { Maternal age at time of } \\
\text { child's birth (years) }\end{array}$} & $<20$ & $508(1.5)$ \\
\hline & $20-24$ & $4436(12.8)$ \\
\hline & $25-29^{*}$ & $8969(25.9)$ \\
\hline & $30-34$ & $12210(35.3)$ \\
\hline & $35-39$ & $7101(20.5)$ \\
\hline & $\geq 40$ & $1350(3.9)$ \\
\hline \multicolumn{3}{|l|}{ Child } \\
\hline \multirow[t]{2}{*}{ Sex } & Female* & $17229(49.8)$ \\
\hline & Male & $17345(50.2)$ \\
\hline \multirow[t]{2}{*}{ Ethnicity } & All other ${ }^{*}$ & $31736(91.8)$ \\
\hline & Indigenous Australian & $2838(8.2)$ \\
\hline \multirow{2}{*}{$\begin{array}{l}\text { Child speaks language } \\
\text { other than English at } \\
\text { home }\end{array}$} & No & $31296(90.5)$ \\
\hline & Yes & $3278(9.5)$ \\
\hline \multirow[t]{3}{*}{$\begin{array}{l}\text { Age category at time of } \\
\text { AEDC collection }\end{array}$} & $\begin{array}{l}\geq 3 \text { years and } 10 \text { months } \\
\text { to }<5 \text { years and } 1 \text { month }\end{array}$ & $5982(17.3)$ \\
\hline & $\begin{array}{l}\geq 5 \text { years and } 1 \text { month to } \\
<5 \text { years and } 10 \text { months }\end{array}$ & $25626(74.1)$ \\
\hline & $\geq 5$ years and 10 months & $2966(8.6)$ \\
\hline
\end{tabular}

Continued 


\begin{tabular}{lll}
\hline Table 1 Continued & & \\
\hline & & Total \\
population \\
$\mathbf{n}(\%)$
\end{tabular}

${ }^{*}$ Reference group for logistic regression.

†Older sibling died within the neonatal period and therefore each cohort child has a valid interpregnancy interval but has no additional siblings born prior to the first year of school.

$\ddagger$ Maternal and paternal occupation status are classified into five categories in line with Australian Socioeconomic Index 2006 (AUSEI06); low AUSEI06 values represent low-status occupations. $\S$ Categorised as nationally defined into five classes of remoteness; $1=$ major cities of Australia (least remote) to $5=$ very remote Australia (most remote).

ๆCategorised as nationally defined quintiles (1=most disadvantaged to $5=$ least disadvantaged); as quintiles are defined nationally (rather than within study population), numbers within each category vary from $20 \%$ of total.

AEDC, Australian Early Development Census. the likelihood of children being classified as DV1 (online supplemental table 1).

\section{Pregnancy and birth variables}

Maternal smoking status during pregnancy, mode of delivery, preterm birth, small for gestational age, parity and maternal age at time of child's birth were all obtained from the MNS and Birth Registrations.

\section{Child variables}

Sex and ethnicity ${ }^{33}$ of child were obtained from the MNS and Birth Registrations. The age of the child at the time of the AEDC collection (reported as a categorical variable) and language other than English spoken at home by the child were obtained from the AEDC. The mean age category for the study population was $\geq 5$ years and 1 month and $<5$ years and 4 months. To balance frequencies, the age of children at the time of AEDC completion was categorised into three groups; (1) $\geq 3$ years and 10 months to $<5$ years and 1 month, (2) $\geq 5$ years and 1 month to $<5$ years and 10 months (reference category) and (3) $\geq 5$ years and 10 months.

\section{Sociodemographic variables}

We derived the total number of siblings by calculating the number of live births each mother had prior to the year that the cohort child had the AEDC conducted for them. Siblings of the cohort who died within the neonatal period were excluded from the calculations. Maternal marital status at time of child's birth was obtained from the MNS and Birth Registrations.

Parental occupational at birth was obtained from Birth Registrations data and converted to a four-digit standard code using the Australian and New Zealand Standard Classification of Occupations. These codes were assigned a value ranging from 0 to 100 in line with the Australian Socioeconomic Index 2006 (AUSEI06). ${ }^{34}$ Low AUSEI06 values represent low-status occupations and high values represent high-status occupations. Records were assigned an AUSEI06 value of zero if occupation was reported as 'unemployed', 'stay at home' parent or 'pensioner.' Cases were classified as missing where parental occupation was not stated. The AUSEI06 values were categorised into five groups: [0, 20], $(20,40],(40,60],(60,80]$ and $(80,100]$.

Remoteness and socioeconomic indices were defined with the Accessibility and Remoteness Index of Australia (ARIA) $^{35}$ and the Index of Relative Socioeconomic Disadvantage (IRSD),${ }^{36}$ respectively, and were calculated using the residential address at the time of birth. The ARIA classifies geographical areas based on access to goods, services and community resources into five categories ranging from; 1 (major cities) to 5 (very remote). The IRSD reflects area-level disadvantage through variables such as low levels of household income, low educational attainment and high levels of unemployment. Geographical areas are given a score from 1 (most disadvantaged) to 5 (most advantaged). 


\section{Multiple imputation}

Overall complete covariate information was available for $86.5 \% \quad(n=29911)$ of the study population. A total of five covariates had missing data; (1) maternal marital status at birth, (2) maternal occupation status scale, (3) paternal occupation status scale, (4) ARIA and (5) IRSD. All variables used in the analysis had $<1.9 \%$ missing data, apart from maternal occupation status $(7.0 \%)$ and paternal occupation status $(7.1 \%)$. Multiple imputation with chained equations, using 20 imputed data sets, was applied to minimise bias attributable to missing data, ${ }^{37}$ and the adjusted analyses presented here were performed by pooling estimates from these imputed data sets.

\section{Sensitivity analysis}

To assess the effect of multiple imputation, we compared the main results of (1) the study based on the imputed data $(n=34574)$ to (2) the results based on the analysis of the complete cases only $(\mathrm{n}=29911$; online supplemental table 2). Although children classified as 'special needs' on the AEDC or with a WARDA record lacked outcome data, it is however, possible that some of the conditions classified as 'special needs' may be related to IPI duration. Therefore, we also conducted a sensitivity analysis that assumed a worst-case scenario, whereby all children with an otherwise complete/valid AEDC record, classified as either 'special needs' on the AEDC or with a WARDA record were classed as developmentally vulnerable (online supplemental table 3; $\mathrm{n}=37789$ ). Finally, to assess whether our results were sensitive to IPI categorisation thresholds, we repeated the analysis by categorising short IPIs as; $<6$ months, $6-11$ months and $12-17$ months,
IPIs of 18-23 months forming the reference category and long IPIs as; 24-41 months, 42-59 months, 60-119 months and $\geq 120$ months (online supplemental table 4).

\section{Statistical modelling}

Logistic regression models were used to estimate the odds of a child being classified as DV1, DV2 or developmentally vulnerable on an individual AEDC domain. Adjustment variables were added simultaneously to the models. ORs and the associated 95\% CIs were estimated for IPIs and adjustment variables. All statistical analyses were conducted in SAS V.9.4. ${ }^{38}$

\section{RESULTS}

\section{Associations between IPIs and developmental vulnerability}

$22.8 \%$ of children were classified as DV1 and $11.5 \%$ were classified as DV2 (table 1). Both unadjusted and adjusted IPIs exhibited J-shaped associations with developmental vulnerability (figure 2 ). In the adjusted models, IPIs of $<6$ months were associated with an increased risk of children being classified as DV1 (adjusted OR (aOR) 1.17, 95\% CI 1.08 to 1.34 ) and DV2 (aOR 1.31, 95\% CI 1.10 to 1.54 ), relative the reference category. All IPIs longer than the reference category were associated with an increased risk of children being classified as DV1; 24-59 months (aOR $1.15,95 \%$ CI 1.05 to 1.25 ), $60-119$ months (aOR 1.43, $95 \%$ CI 1.28 to 1.60 ) and $\geq 120$ months (aOR $1.84,95 \%$ CI 1.54 to 2.19 ), and DV2; $24-59$ months (aOR 1.19 , 95\% CI 1.06 to 1.34 ), $60-119$ months (aOR $1.55,95 \%$ CI 1.35 to 1.79 ) and $\geq 120$ months (aOR $1.78,95 \%$ CI 1.42 to 2.24 ).
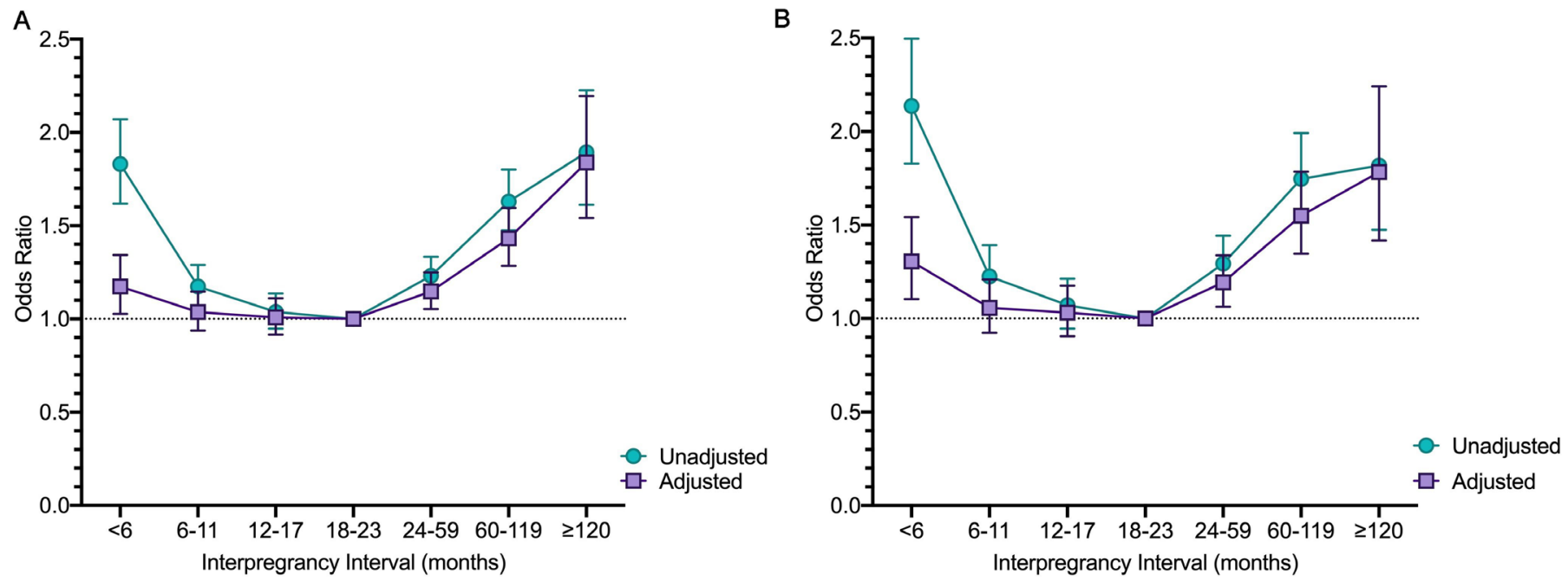

Figure 2 Unadjusted and adjusted ORs for the association between interpregnancy intervals (IPIs) and developmental vulnerability on Australian Early Development Census (AEDC) domains. ORs relative to the IPI interval reference category of 18-23 months between IPI and (A) developmental vulnerability on one or more AEDC domains (DV1) and (B) developmental vulnerability on two or more AEDC domains (DV2). Adjusted for maternal smoking status during pregnancy, mode of delivery, preterm birth, small for gestational age, parity, mother's age at time of child's birth, sex of child, ethnicity, child speaks a language other than English at home, age of child at time of AEDC completion, number of siblings, mother's marital status at time of child's birth, father's and mother's occupational status scale at time of child's birth, Accessibility and Remoteness Index of Australia and Index of Relative Socioeconomic Disadvantage category. All data is presented as ORs and 95\% Cls; logistic regression $(n=34574)$. 
Associations between IPIs and domain-specific developmental vulnerability

A total of $3691(10.7 \%)$ children were classified as developmentally vulnerable for the domains of; Physical Health and Wellbeing, 2794 (8.1\%) for Social Competence, 2817 $(8.1 \%)$ for Emotional Maturity, 3381 (9.8\%) for Language and Cognitive Skills (school-based) and 2856 (8.3\%) for Communication Skills and General Knowledge (table 2). In the adjusted models, IPIs of $<6$ months were associated with an increased risk of developmental vulnerability for the domains of Physical Health and Wellbeing (aOR 1.25, 95\% CI 1.06 to 1.48; table 2) and Emotional Maturity (aOR $1.36,95 \%$ CI 1.12 to 1.66 ) and IPIs of 6-11 months were associated with an increased risk of developmental vulnerability on the domain of Emotional Maturity (aOR 1.27, 95\% CI 1.09 to 1.48 ), only. IPIs of $60-119$ and $\geq 120$ months were associated with an increased risk of developmental vulnerability on each of the five AEDC domains, with greater odds for each domain for the longer IPI category. IPIs of 24-59 months were associated with an increased risk of developmental vulnerability for all AEDC domains, except Physical Health and Wellbeing, and Communication Skills and General Knowledge.

\section{Sensitivity analysis}

Sensitivity analysis revealed that the overall associations between IPIs and developmental vulnerability at age 5 were not substantially different between complete cases and the imputed cases (online supplemental table 2).

\section{DISCUSSION}

We found that short IPIs of $<6$ months and all longer IPIs ( $\geq 24$ months) were associated with increased odds of developmental vulnerability, compared with an IPI of 18-23 months. These results were obtained from a large population of $>34000$ children and associations were not fully explained by pregnancy, birth, child and sociodemographic characteristics. Thus, the results indicate the potential for the adverse effects of short and longer IPIs to persist beyond the perinatal period and into early childhood.

Few studies have examined the associations between pregnancy spacing and school readiness or academic performance. ${ }^{1620}$ A US study of 5339 children using data from the National Longitudinal Survey of Youth 1979 (NLSY79) assessed the associations between IPIs and child cognitive ability and externalising behavioural symptoms (as assessed by the Behaviour Problem Index) in early and middle childhood. ${ }^{19}$ This study reported that after controlling for the sex of the child and birth order, short IPIs ( $\leq 12$ months) were associated with scores lower than the mean for performance in the Peabody Picture Vocabulary Test and the maths, reading and reading recognition subtests of the Peabody Individual Achievement Test-Revised (PIAT-R) ${ }^{19}$ Furthermore, this study also reported that long IPIs (>36 months) were associated with scores lower than the mean on the maths component of the PIAT-R. ${ }^{19}$ However, after controlling for several additional factors including, maternal age, ethnicity and family income this study reported no statistically significant association between suboptimal IPI duration and child development outcomes. ${ }^{19}$ Furthermore, this study reported no statistically significant associations between short or long IPIs and externalising behavioural problems in children. ${ }^{19}$ Our results also indicate that very short IPIs $(<6$ months) and long IPIs ( $\geq 24$ months) are associated with increased odds of developmental vulnerability in the adjusted models. Likewise, a cohort study of 6915 children from South Carolina (USA) concluded that children born after short birth intervals ( $<24$ months) were more likely to fail a school readiness test when compared with children born with longer birth intervals (24-120 months), after controlling for maternal risk factors including education level, ethnicity and marital status. ${ }^{16}$ Our results also indicate that very short IPIs ( $<6$ months) are associated with increased odds of developmental vulnerability. However, we also reported that IPIs $\geq 24$ months were associated with developmental vulnerability.

Differences in findings between these studies and our study may be attributed to differences in the definition of the reference categories. The birth interval reference category of 24-120 months used in the South Carolina study would equate to an IPI of roughly 15-111 months (based on a term pregnancy), while the reference category for the NLSY79 study was 12-36 months. These reference categories are wide and overlaps with several of the IPI categories used in our study, for which the direction of associations were not consistent. ${ }^{16}$ Taken together the results of both US studies and our study provide preliminary evidence to suggest that short IPIs may be associated with an increased likelihood of developmental vulnerability in children and that the effects of the maternal depletion hypothesis may extend beyond birth outcomes. It is estimated that approximately half of the pregnancies in Australia are unplanned. ${ }^{39}$ Decreasing the frequency of suboptimal IPIs may improve birth outcomes and as a result, may further improve overall school readiness in the population. However, further research is needed to establish if this relationship is causal.

Alternatively, a longitudinal study of 1154 French children, assessing the relationship between language skills of children aged between 5 and 6 years of age and the age gap between their immediately older sibling, reported that more closely spaced siblings were more likely to have higher language scores. ${ }^{17}$ The results of our study however, reported an increased, although statistically insignificant odds of developmental vulnerability the AEDC domains of Language and Cognitive Skills (schoolbased) and Communication Skills and General Knowledge. It should be noted that the French study modelled age gap as a continuous variable and thus this may account for variations in the findings of the association between birth spacing and child development outcomes between this study and our study. Furthermore, the French study concluded that a larger study would be required to determine if the negative age-gap effect was genuine. 


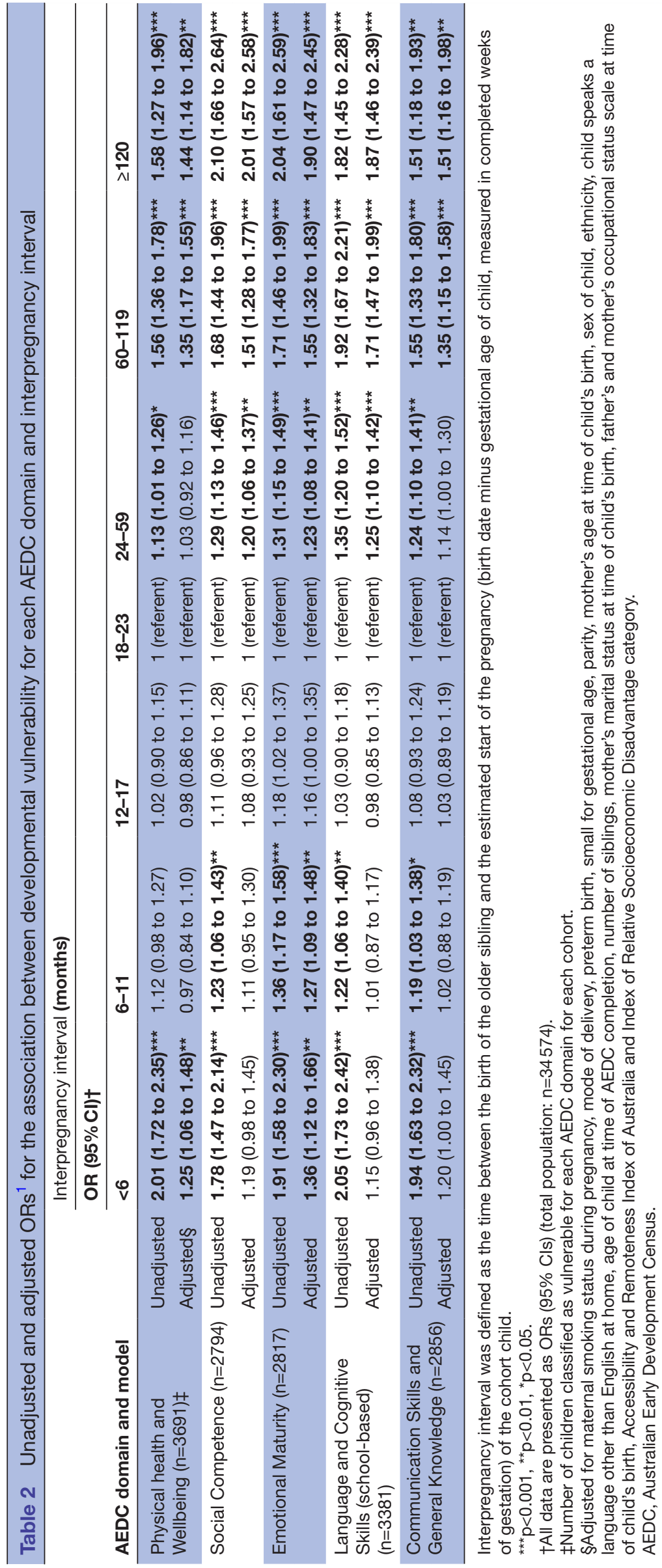


The available studies assessing associations between birth spacing and academic performance have been primarily conducted in children older than those assessed in our study. One of the earliest studies to investigate the relationship between school performance conducted in Singapore reported that children born after a birth interval of $\geq 24$ months performed better at school, at age 9 than children born after a birth interval of $<24$ months. ${ }^{20}$ Alternatively, a cross-sectional study of Saudi boys, aged between 9 and 10 years, reported that children born after short birth intervals of $<17$ months were more likely to be classified as below average on teacherassessed school performance in comparison to children born after long birth intervals ( $>31$ months).${ }^{14}$ Again, differences in findings between these studies and those of our study may be attributed to the fact that both of these studies used binary birth interval categories, which overlap with several of the IPI categories used in our study and with the current WHO recommendations of an IPI of approximately 2-3 years. ${ }^{40}$ Given these mixed results and the relatively small number of studies on the topic, the association between IPIs and developmental vulnerability beyond the perinatal period remains not well-established. Furthermore, as prior research assessing the associations between IPIs and child development outcomes has largely been confined to the perinatal period, further research is required to assess the relationship between IPIs and developmental vulnerability in early childhood and to determine causality.

\section{Limitations}

Our study had several limitations. First, important social risk factors including parenting experience and/or technique, stability and quality of housing and availability of learning resources within the household could not be accounted for due to the nature of administrative data. Second, we did not have information as to whether the pregnancies were planned or unplanned and as administrative records do not include pregnancies ending before 20 weeks of gestations, we are unable to identify and account for the effect of miscarriages. Third, as the birthdates of older siblings were obtained from Birth Registrations and MNS records in the state of WA, we were not able to calculate IPIs for children with an older sibling born in another state. Finally, there are a wide range of reproductive health behaviours and physiological factors governing the length of IPIs including, fertility levels, the use of contraception, time-until-conception and breastfeeding durations, ${ }^{41}$ which we could not control for as they are not recorded in administrative data.

\section{Implications of findings}

The behaviours, emotions and physical and cognitive capacities that develop in the first 5 years of life assist in the facilitation of learning and are predictive of later school achievement and behavioural outcomes. ${ }^{25-27}$ The cumulative nature of school-based learning means that children who begin school with poor school readiness often fail to catch up with their peers and tend to fall further behind as they progress through schooling. ${ }^{28}$ In particular, research has indicated that children classified as DV1 are more likely to score in the bottom $20 \%$ of all students on the National Assessment Program Literacy and Numeracy assessments in grades 3, 5 and $7 .{ }^{42}$ This study supports the hypothesis that IPIs are a predictor of developmental vulnerability and poor school readiness. The concept of optimising birth spacing has been widely discussed in the literature, and the findings of our study imply that the adverse impacts of IPIs may extend beyond birth outcomes. Decreasing the frequency of suboptimal IPIs may improve birth outcomes and as a result, may further improve overall child development outcomes in the population.

\section{CONCLUSIONS}

IPIs exhibited independent J-shaped associations with developmental vulnerability. For our cohort, very short IPIs of less than 6 months and longer IPIs of 2 years or longer were associated with increased risk of developmental vulnerability on one or more and two or more AEDC domains. IPIs of 5 years or longer were associated with developmental vulnerability on all five AEDC domains. The results provide empirical support for the association between long IPIs of 2 years or longer and developmental vulnerability at age 5 . Although further research is required to establish causality, the results of this study add to the current body of literature suggesting the potential for optimising IPIs as a means for improving child development outcomes.

Acknowledgements We gratefully acknowledge the WA Data Linkage Branch and Data Custodians who provided data for this study and the people of Western Australia for the use of their administrative data. This study does not necessarily reflect their views. This study uses data from the Australian Early Development Census (AEDC). The AEDC is funded by the Australian Government Department of Education and Training. The findings and views reported are those of the authors and should not be attributed to the Department or the Australian Government. We thank Helen Bailey for assistance in the use of these data and Scott Sims and Daniel Christensen for statistical advice and support.

Contributors GKD led study conceptualisation and design, conducted the literature review, performed data manipulation, analysis and interpretation of findings, drafted the initial manuscript and reviewed and revised the manuscript critically for important intellectual content. GP and CLT contributed to conceptualising and designing the study, interpreting the results and writing the manuscript. All authors approved the final manuscript as submitted and agree to be accountable for all aspects of the work.

Funding This work was supported by the National Health and Medical Research Grants (grant numbers GNT1173991 and GNT1099655 to GP), the Australian Research Council Centre of Excellence for Children and Families over the Life Course (grant number CE140100027 to CLT). GKD was supported by the ARC Centre of Excellence for Children and Families over the Life Course Scholarship, the ARC Centre of Excellence for Children and Families over the Life Course Top-Up Scholarship and the Stan and Jean Perron Top-Up Scholarship. GP was supported with funding from the National Health and Medical Research Council Project and Investigator (grant numbers 1099655 and 1173991) and the Research Council of Norway through its Centres of Excellence funding scheme (grant number 262700).

Competing interests None declared.

Patient consent for publication Not required.

Ethics approval Ethics approval for this study was granted by the Western Australian Department of Health Human Research Ethics Committee (2016/51) 
and the University of Western Australia Human Research Ethics Committee (RA/4/20/4776)

Provenance and peer review Not commissioned; externally peer reviewed.

Data availability statement All data relevant to the study are included in the article or uploaded as supplementary information. We received data from WA Department of Health through the Data Linkage Branch. The data are not publicly available, and privacy and legal restrictions apply to the provision of the data to third parties.

Supplemental material This content has been supplied by the author(s). It has not been vetted by BMJ Publishing Group Limited (BMJ) and may not have been peer-reviewed. Any opinions or recommendations discussed are solely those of the author(s) and are not endorsed by BMJ. BMJ disclaims all liability and responsibility arising from any reliance placed on the content. Where the content includes any translated material, BMJ does not warrant the accuracy and reliability of the translations (including but not limited to local regulations, clinical guidelines, terminology, drug names and drug dosages), and is not responsible for any error and/or omissions arising from translation and adaptation or otherwise.

Open access This is an open access article distributed in accordance with the Creative Commons Attribution Non Commercial (CC BY-NC 4.0) license, which permits others to distribute, remix, adapt, build upon this work non-commercially, and license their derivative works on different terms, provided the original work is properly cited, appropriate credit is given, any changes made indicated, and the use is non-commercial. See: http://creativecommons.org/licenses/by-nc/4.0/.

\section{ORCID iDs}

Gursimran Kaur Dhamrait http://orcid.org/0000-0002-5191-211X

Catherine Louise Taylor http://orcid.org/0000-0001-9061-9162

Gavin Pereira http://orcid.org/0000-0003-3740-8117

\section{REFERENCES}

1 Ball SJ, Pereira G, Jacoby P, et al. Re-evaluation of link between interpregnancy interval and adverse birth outcomes: retrospective cohort study matching two intervals per mother. BMJ 2014;349:g4333.

2 Shachar BZ, Lyell DJ. Interpregnancy interval and obstetrical complications. Obstet Gynecol Surv 2012;67:584-96.

3 Grisaru-Granovsky S, Gordon E-S, Haklai Z, et al. Effect of interpregnancy interval on adverse perinatal outcomes-a national study. Contraception 2009;80:512-8.

4 Conde-Agudelo A, Belizán JM, Norton MH, et al. Effect of the interpregnancy interval on perinatal outcomes in Latin America. Obstet Gynecol 2005;106:359-66.

5 Hanley GE, Hutcheon JA, Kinniburgh BA, et al. Interpregnancy interval and adverse pregnancy outcomes: an analysis of successive pregnancies. Obstet Gynecol 2017;129:408-15.

6 Conde-Agudelo A, Rosas-Bermudez A, Norton MH. Birth spacing and risk of autism and other neurodevelopmental disabilities: a systematic review. Pediatrics 2016;137 doi:10.1542/peds.20153482

7 Zhu B-P. Effect of interpregnancy interval on birth outcomes: findings from three recent US studies. Int J Gynaecol Obstet 2005;89:S25-33.

8 Miller JE. Birth intervals and perinatal health: an investigation of three hypotheses. Fam Plann Perspect 1991;23:62-70.

9 Habimana-Kabano I, Broekhuis A, Hooimeijer P. The effects of Interpregnancy intervals and previous pregnancy outcome on fetal loss in Rwanda (1996-2010). Int J Reprod Med 2015;2015:10.

10 Carlsson T, Molander F, Taylor MJ, et al. Early environmental risk factors for neurodevelopmental disorders - a systematic review of twin and sibling studies. Dev Psychopathol 2020:1-48.

11 Elhakham D, Wainstock T, Sheiner E, et al. Inter-pregnancy interval and long-term neurological morbidity of the offspring. Arch Gynecol Obstet 2020 doi:10.1007/s00404-020-05788-9

12 Cheslack-Postava K, Sourander A, Suominen A. Increased risk of $\mathrm{ADHD}$ at short and long Interpregnancy intervals in a national birth cohort. Paediatr Perinat Epidemiol 2020

13 Class QA, Rickert ME, Oberg AS, et al. Within-family analysis of Interpregnancy interval and adverse birth outcomes. Obstet Gynecol 2017;130:1304-11.

14 Bella H, Al-Almaie SM. Do children born before and after adequate birth intervals do better at school? J Trop Pediatr 2005;51:265-70.

15 Bella H, Khalil MS, Al-Almaie SM, et al. The effects of birth interval on intellectual development of Saudi school children in eastern Saudi Arabia. Saudi Med J 2005;26:741-5.
16 Hayes H, Luchok K, Martin AB, et al. Short birth intervals and the risk of school unreadiness among a Medicaid population in South Carolina. Child Care Health Dev 2006;32:423-30.

17 Havron N, Ramus F, Heude B, et al. The effect of older siblings on language development as a function of age difference and sex. Psychol Sci 2019;30:1333-43.

18 Nathens AB, Neff MJ, Goss CH, et al. Effect of an older sibling and birth interval on the risk of childhood injury. Inj Prev 2000;6:219.

19 Sujan AC, Class QA, Rickert ME, et al. Risk factors and child outcomes associated with short and long interpregnancy intervals. Early Child Dev Care 2019;34:1-12.

20 Martin C. A study of influences on birth interval in Singapore. J Trop Pediatr Env Child Health 1979;25:49-76.

21 Cofer FG, Fridman M, Lawton E, et al. Interpregnancy interval and childbirth outcomes in California, 2007-2009. Matern Child Health J 2016;20:43-51.

22 Guhn M, Gadermann AM, Almas A, et al. Associations of teacherrated social, emotional, and cognitive development in kindergarten to self-reported wellbeing, peer relations, and academic test scores in middle childhood. Early Child Res Q 2016;35:76-84.

23 Domitrovich C, Moore J, Thompson R. Interventions that promote social-emotional learning in young children. In: Pianta RC, Barnett SW, Justice LM, eds. Handbook of early childhood education. New York, NY: Guilford Press, 2012: 393-415.

24 Holman C D'Arcy J, Bass AJ, Rosman DL, et al. A decade of data linkage in Western Australia: strategic design, applications and benefits of the WA data linkage system. Aust Health Rev 2008;32:766-77.

25 Guthridge S, Li L, Silburn S, et al. Early influences on developmental outcomes among children, at age 5, in Australia's Northern Territory. Early Child Res Q 2016;35:124-34.

26 Australian Government Department of Education \& Training. Australian Early Development Census (AEDC) data guidelines department of education \& training 2017.

27 Department of Education Employment and Workplace Relations for the Council of Australian Governments (DEEWR). Belonging, being and becoming: the early years learning framework for Australia. Canberra: DEEWR, 2009.

28 Department of Education and Training. Australian early development census national report 2015. Canberra: Department of Education and Training, 2016

29 Chen X-K, Wen SW, Fleming N, et al. Teenage pregnancy and adverse birth outcomes: a large population based retrospective cohort study. Int J Epidemiol 2007;36:368-73.

30 Crosnoe R, Wildsmith E. Nonmarital fertility, family structure, and the early school achievement of young children from different Race/ Ethnic and immigration groups. Appl Dev Sci 2011;15:156-70.

31 Boyle MH, Georgiades K, Racine Y, et al. Neighborhood and family influences on educational attainment: results from the Ontario child health study follow-up 2001. Child Dev 2007;78:168-89.

32 Dhamrait GK, Christensen D, Pereira G, et al. Associations between biological and sociodemographic risks for developmental vulnerability in twins at age 5: a population data linkage study in Western Australia. BMJ Open 2020;10:e038846.

33 Christensen D, Davis G, Draper G, et al. Evidence for the use of an algorithm in resolving inconsistent and missing Indigenous status in administrative data collections. Aust J Soc Issues 2014;49:423-43.

34 McMillan J, Beavis A, Jones F. The AUSEI06:A new socioeconomic index for Australia. J Sociol 2009;45:123-49.

35 Australian Bureau of Statistics. The Australian statistical geography standard (ASGS) remoteness structure, 2018. Available: https://www. abs.gov.au/websitedbs/D3310114.nsf/home/remoteness+structure [Accessed 10 Sep 2019].

36 Australian Bureau of Statistics. Index of relative socio-economic disadvantage, 2018. Available: https://www.abs.gov.au/ausstats/ abs@.nsf/Lookup/by\%20Subject/2033.0.55.001 2016 Main\% 20Features IRSD 19 [Accessed 10 Sep 2019].

37 Royston P, White I. Multiple Imputation by Chained Equations (MICE): Implementation in Stata. J Stat Softw 2011;45:1-20.

38 SAS Institute. SAS (program). Cary, NC: SAS Institute, 2013.

39 Marie Stopes International Australia. Real choices: women, contraception and unplanned pregnancy. Melbourne: Marie Stopes International Australia, 2008.

40 World Health Organization. Report of a technical consultation on birth spacing. Geneva: WHO, 2005.

41 Wendt A, Gibbs CM, Peters S, et al. Impact of increasing interpregnancy interval on maternal and infant health. Paediatr Perinat Epidemiol 2012;26 Suppl 1:239-58.

42 Brinkman S, Gregory T, Harris J, et al. Associations between the early development instrument at age 5 , and reading and Numeracy skills 
